\title{
AN ESTIMATE FOR THE RATE OF CONVERGENCE OF THE DISTRIBUTION OF THE NUMBER OF FALSE SOLUTIONS OF A SYSTEM OF NONLINEAR RANDOM EQUATIONS IN THE FIELD $G F(2)$
}

UDC 519.21

\author{
V. I. MASOL AND M. V. SLOBODYAN
}

ABSTRACT. We prove a result on the rate of convergence as $n \rightarrow \infty$ of the distribution of the number of false solutions of a system of nonlinear random equations in the field $G F(2)$ to the Poisson distribution with parameter $2^{m}$. We assume, in particular, that the difference between the number $n$ of unknowns and the number $N$ of equations of the system is a constant $m$.

\section{Setting of the Problem. Statement of the Result}

Consider the following system of equations:

$$
\sum_{k=1}^{g_{i}(n)} \sum_{1 \leq j_{1}<\cdots<j_{k} \leq n} a_{j_{1} \ldots j_{k}}^{(i)} x_{j_{1}} \cdots x_{j_{k}}=b_{i}, \quad i=1,2, \ldots, N,
$$

in the field $G F(2)$. Throughout the paper we assume that the following conditions are satisfied:

- the coefficients $a_{j_{1} \ldots j_{k}}^{(i)}, 1 \leq j_{1}<\cdots<j_{k} \leq n, k=1,2, \ldots, g_{i}(n), i=1,2, \ldots, N$, are independent random variables such that

$$
\mathrm{P}\left\{a_{j_{1} \ldots j_{k}}^{(i)}=1\right\}=1-\mathrm{P}\left\{a_{j_{1} \ldots j_{k}}^{(i)}=0\right\}=p_{i k} ;
$$

- the elements $b_{i}, i=1,2, \ldots, N$, are obtained after the substitution of a fixed $n$-dimensional $(0,1)$-vector $\bar{x}^{0}$ with exactly $\rho(n)$ nonzero coordinates to the left hand side of system (11) where

$$
\rho(n)=\rho n, \quad \rho=\text { const }, \quad 0<\rho<1 ;
$$

- the functions $g_{i}(n)$ are nonrandom, $g_{i}(n) \in\{2,3, \ldots, n\}, i=1,2, \ldots, N$.

We denote this set of conditions by (A).

Let $\nu_{n}$ be the total number of false solutions of system (1), that is, the total number of solutions of system (11) that do not coincide with $\bar{x}^{0}$. In this paper, we study the rate of convergence of the distribution of the random variable $\nu_{n}$ to the limit Poisson distribution with parameter $2^{m}$ if condition (2) holds.

Theorem. Suppose the conditions (A) are satisfied. Assume that

$$
n-N=m, \quad m=\text { const }, \quad-\infty<m<\infty,
$$

2000 Mathematics Subject Classification. Primary 60C05, 15A52, 15A03.

Key words and phrases. System of nonlinear random equations, the field $G F(2)$, rate of convergence. 
and that for any $i=1,2, \ldots, N$, there exists a set $T_{i} \neq \varnothing$ such that

$$
T_{i} \subseteq\left\{2, \ldots, g_{i}(n)\right\}, \quad 0 \leq \delta_{i t}(n) \leq p_{i t} \leq 1-\delta_{i t}(n), t \in T_{i},
$$

for sufficiently large $n$, where $\delta_{i t}(n)$ are some numbers such that $0 \leq \delta_{i t}(n) \leq \frac{1}{2}$. Furthermore, let a function $\varphi(n)$ be such that $\varphi(n) \leq \ln ^{2} n$. Assume that, given a constant $\varepsilon_{0} \in(0 ; 1)$ and a fixed integer $l \geq 0$, one can find a natural number $n_{0}=n_{0}\left(\varepsilon_{0}, l\right)$ for which

$$
2^{\gamma+1} B(n)<\varepsilon_{0}
$$

for all $n \geq n_{0}$, where $\gamma=\left[\log _{2} n / 6\right]$ for $n \geq 2^{6 l}$,

$$
B(n)=\sum_{i=1}^{N} \exp \left\{-2 \sum_{t \in T_{i}} \delta_{i t}(n) C_{f(n)}^{t}\right\},
$$

and $f(n)$ assumes positive integer values and is such that $f(n)=o(\varphi(n))$ as $n \rightarrow \infty$. (Here and in what follows, the symbol $C_{f(n)}^{t}$ stands for the binomial coefficient $\left(\begin{array}{c}f(n) \\ t\end{array}\right)$.) If $k=0,1,2, \ldots$ is fixed, then

$$
\begin{aligned}
\left|\mathrm{P}\left\{\nu_{n}=k\right\}-\frac{\lambda^{k}}{k !} e^{-\lambda}\right| \leq & \left(\frac{2 e \lambda}{\gamma}\right)^{\gamma}\left\{2+2^{\gamma+1} B(n)+\Theta_{2}\left(1+2^{\gamma+1} B(n)\right)+7 \Theta_{1}\right\} \\
& +e^{4 \lambda} \gamma B(n)+e^{2 \lambda} \gamma\left(\Theta_{2}\left(1+2^{\gamma+1} B(n)\right)+7 \Theta_{1}\right),
\end{aligned}
$$

where $\lambda=2^{m}$,

$$
\begin{gathered}
\Theta_{1}=\exp \left\{-2^{-2 \gamma} \sum_{i=1}^{N} \delta_{i}+\log _{2} \sqrt[6]{n}+\sqrt[6]{n}+\ln (\tilde{\rho} n)-m \ln 2\right\}, \\
\Theta_{2}=2^{-n} \exp \left\{\varepsilon \sqrt[6]{n} \ln ^{2} n\left(\log _{2} \sqrt[6]{n}+\ln \left(\frac{n^{5 / 6} e}{\varepsilon \ln ^{2} n}\right)\right)+\sqrt[6]{n}+2 \ln \left(\varepsilon \sqrt[6]{n} \ln ^{2} n\right)\right\}, \\
\tilde{\rho}=\max \{\rho, 1-\rho\}, \quad \delta_{i}=\min \left\{\sum_{t \in T_{i}} \delta_{i t}(n) C_{r}^{t-1}, \frac{2 \ln n}{\sqrt{\varepsilon} \varphi(n)}\right\},
\end{gathered}
$$

$r=[\varepsilon \varphi(n)], 0<\varepsilon<1$, and $\varepsilon=$ const.

Here and in what follows we assume that $0^{0} \equiv 1$.

Remark. Fix arbitrary numbers $\varepsilon_{0} \in(0 ; 1)$ and $\varepsilon \in(0 ; 1)$. It is easy to check that, given a number $\gamma>0$, there exists a natural number $n_{1}=n_{1}\left(\varepsilon_{0}, \varepsilon, \gamma\right)$ such that the right hand side of inequality (6) becomes smaller than $\gamma$ for all $n \geq n_{1}$.

Example. If $T_{i}=\{2\}, \varphi(n)=\ln ^{2} n, f(n)=\left[(\ln n)^{3 / 2}\right], \delta_{i t}=\frac{1}{2}, n=65, m=-8$, $\rho=0.9, \varepsilon=0.25$, and $\varepsilon_{0} \leq \varepsilon$, then relations (3)-(5) hold. Applying inequality (6) we obtain

$$
\left|\mathrm{P}\left\{\nu_{65}=k\right\}-\frac{\lambda^{k}}{k !} e^{-\lambda}\right| \leq 0.086
$$

for all $k \geq 0$.

\section{Auxiliary Results}

Denote by $\mathrm{E} \nu_{n}^{[k]}$ the factorial moment of order $k$ for the random variable $\nu_{n}, k=$ $1,2, \ldots$ We set $\mathrm{E} \nu_{n}^{[0]} \equiv 1$. 
Proposition ([1]). If the conditions (A) hold, then, for all $k \geq 1$,

$$
\mathrm{E} \nu_{n}^{[k]}=2^{-k N} S(n, k ; Q)
$$

where

$$
\begin{aligned}
S(n, k ; Q)= & \sum_{s=0}^{n-\rho n} \sum(n-\rho n) !\left((n-\rho n-s) ! \prod_{i \in I} i !\right)^{-1} \\
& \times \sum_{\substack{s^{\prime}=0 \\
s^{\prime}+s \geq 1}}^{\rho n} \sum^{\prime}(\rho n) !\left(\left(\rho n-s^{\prime}\right) ! \prod_{j \in J} j !\right)^{-1} Q \\
Q= & \prod_{i=1}^{N}\left(1+\sum_{\nu=1}^{k} \sum_{1 \leq u_{1}<\cdots<u_{\nu} \leq k} \prod_{t=1}^{g_{i}(n)}\left(1-2 p_{i t}\right)^{\Gamma_{t, k}^{\left\{u_{1}, \ldots, u_{\nu}\right\}}}\right)
\end{aligned}
$$

and the index of summation in $\sum\left(\sum^{\prime}\right)$ runs over all elements $i \in I(j \in J)$ such that

$$
\sum_{i \in I} i=s, \quad \sum_{j \in J} j=s^{\prime}
$$

where

$$
\begin{aligned}
& I=\left\{i_{\left\{u_{1}, \ldots, u_{\nu}\right\}}: 1 \leq u_{1}<\cdots<u_{\nu} \leq k, \nu=1, \ldots, k\right\}, \\
& J=\left\{j_{\left\{u_{1}, \ldots, u_{\nu}\right\}}: 1 \leq u_{1}<\cdots<u_{\nu} \leq k, \nu=1, \ldots, k\right\} .
\end{aligned}
$$

(The definition of the numbers $i_{\left\{u_{1}, \ldots, u_{\nu}\right\}}$ and $j_{\left\{u_{1}, \ldots, u_{\nu}\right\}}$ is given in 1].) The elements $i \in I$ and $j \in J$ in inequality (8) are such that

$$
\sum_{\substack{i \in I_{\{u\}}, j \in J_{\{u\}}}}(i+j) \geq 1, \quad u=1, \ldots, k,
$$

(the sets $I_{\{u\}}$ and $J_{\{u\}}$ are defined below) and

$$
\begin{gathered}
\sum_{l=0}^{k-2} \sum_{1 \leq \mu_{1}<\cdots<\mu_{l} \leq k}\left(i_{\left\{u_{1}, \mu_{1}, \ldots, \mu_{l}\right\}}+j_{\left\{u_{1}, \mu_{1}, \ldots, \mu_{l}\right\}}+i_{\left\{u_{2}, \mu_{1}, \ldots, \mu_{l}\right\}}+j_{\left\{u_{2}, \mu_{1}, \ldots, \mu_{l}\right\}}\right) \geq 1, \\
1 \leq u_{1}<u_{2} \leq k,
\end{gathered}
$$

for $1 \leq u_{1}<\cdots<u_{\nu} \leq k, \nu \in\{1, \ldots, k\}$, and $t \in\{1, \ldots, n\}$. Moreover

$$
\Gamma_{t, k}^{\left\{u_{1}, \ldots, u_{\nu}\right\}} \geq \sum_{(i, j) \in T}\left(C_{i}^{t}+C_{j}^{t}\right)
$$

where

Here

$$
T=I_{\left\{u_{1}, \ldots, u_{\nu}\right\}} \times J_{\left\{u_{1}, \ldots, u_{\nu}\right\}} .
$$

$$
\begin{aligned}
& I_{\left\{u_{1}, \ldots, u_{\nu}\right\}}=\left\{i_{\left\{\sigma_{1}, \ldots, \sigma_{\psi}, \mu_{1}, \ldots, \mu_{l}\right\}}: A(\psi, l, k)\right\}, \\
& J_{\left\{u_{1}, \ldots, u_{\nu}\right\}}=\left\{j_{\left\{\sigma_{1}, \ldots, \sigma_{\psi}, \mu_{1}, \ldots, \mu_{l}\right\}}: A(\psi, l, k)\right\}
\end{aligned}
$$

are the sets of numbers $i_{\left\{\sigma_{1}, \ldots, \sigma_{\psi}, \mu_{1}, \ldots, \mu_{l}\right\}}$ and $j_{\left\{\sigma_{1}, \ldots, \sigma_{\psi}, \mu_{1}, \ldots, \mu_{l}\right\}}$, respectively, satisfying the collection of restrictions $A(\psi, l, k)$, where $A(\psi, l, k)$ means

$$
\begin{gathered}
1 \leq \sigma_{1}<\cdots<\sigma_{\psi} \leq k, \quad \sigma_{z} \in\left\{u_{1}, \ldots, u_{\nu}\right\}, \quad z=1, \ldots, \psi, \quad \psi=1, \ldots, \nu \\
\psi \equiv 1(\bmod 2), \quad 1 \leq \mu_{1}<\cdots<\mu_{l} \leq k, \quad \mu_{1}, \ldots, \mu_{l} \notin\left\{u_{1}, \ldots, u_{\nu}\right\} \\
l=0, \ldots, k-\nu .
\end{gathered}
$$

If

$$
\rho n-s^{\prime} \geq t,
$$


then

$$
\Gamma_{t, k}^{\left\{u_{1}, \ldots, u_{\nu}\right\}} \geq C_{\rho n-s^{\prime}}^{t-1} \sum_{(i, j) \in T}(i+j) .
$$

The explicit expression for $\Gamma_{t, k}^{\left\{u_{1}, \ldots, u_{\nu}\right\}}$ is given in [1] for the case of $1 \leq u_{1}<\cdots<$ $u_{\nu} \leq k, \nu \in\{1, \ldots, k\}, t=1,2, \ldots, g_{i}(n), i=1, \ldots, N$.

To prove the theorem of Section 1, we need the following auxiliary result.

Lemma. Suppose all the assumptions of the theorem hold for all nonnegative integers $k$ such that

$$
0<k \leq \gamma
$$

Then

$$
\mathrm{E} \nu_{n}^{[k]}=\lambda^{k}+\Delta(k, n)
$$

for all sufficiently large $n$, where

$$
\begin{gathered}
|\Delta(k, n)| \leq 2^{(m+1) k+1} u+2^{m k} \Theta_{2}\left(1+2^{k+1} u\right)+7\left(2^{2^{k}}\right) 2^{(m+1) k} \\
\times \exp \left\{-2^{-2 k} \sum_{i=1}^{N} \delta_{i}+\ln (\tilde{\rho} n)-m \ln 2\right\}, \\
u=\sum_{i=1}^{N} \exp \left\{-2 \sum_{t \in T_{i}} \delta_{i t}(n) C_{r}^{t}\right\} .
\end{gathered}
$$

Proof. Using equality (7), we represent the factorial moment $\mathrm{E} \nu_{n}^{[k]}$ as follows:

$$
\mathrm{E} \nu_{n}^{[k]}=2^{-k N} \sum_{\Delta \geq 0} S^{(\Delta)}(n, k ; Q),
$$

where $S^{(\Delta)}(n, k ; Q)$ is defined similarly to the term $S(n, k ; Q)$ with additional restrictions imposed on elements $i \in I$ and $j \in J$ appearing in definition (8) of $S(n, k ; Q)$, namely, that there are exactly $\Delta$ pairwise distinct sets $\omega_{\alpha}$,

$$
\begin{gathered}
\omega_{\alpha}=\left\{u_{1}^{(\alpha)}, \ldots, u_{\xi_{\alpha}}^{(\alpha)}\right\}, \\
1 \leq u_{1}^{(\alpha)}<\cdots<u_{\xi_{\alpha}}^{(\alpha)} \leq k, \quad \xi_{\alpha} \in\{1, \ldots, k\}, \quad \alpha=1, \ldots, \Delta,
\end{gathered}
$$

such that for each of them there exists $t^{(\alpha)} \in\{2, \ldots, r\}$ that satisfies

$$
\Gamma_{t^{(\alpha)}, k}^{\omega_{\alpha}}<C_{r}^{t^{(\alpha)}}
$$

and

$$
\Gamma_{t, k}^{\left\{v_{1}, \ldots, v_{\gamma}\right\}} \geq C_{r}^{t}
$$

for all $t \in\{2, \ldots, r\}$ and for all sets $\left\{v_{1}, \ldots, v_{\gamma}\right\}, 1 \leq v_{1}<\cdots<v_{\gamma} \leq k, \gamma=1, \ldots, k$, such that $\left\{v_{1}, \ldots, v_{\gamma}\right\} \neq \omega_{\alpha}, \alpha=1, \ldots, \Delta$.

It is worth mentioning that the term corresponding to $\Delta=0$ may indeed appear on the right hand side of (15) (see [1]).

Furthermore, we rewrite equality (15) as follows:

$$
\mathrm{E} \nu_{n}^{[k]}=S_{1}+p_{1},
$$

where

$$
S_{1}=2^{-k N} S^{(0)}(n, k ; Q), \quad p_{1}=2^{-k N} \sum_{\Delta=1}^{2^{k}-1} S^{(\Delta)}(n, k ; Q) .
$$


Now we turn to the estimation of $S_{1}$. If $\Delta=0$, we use estimate (17) and condition (4). Then

$$
\begin{aligned}
& \prod_{i=1}^{N}\left(1-2^{k} \exp \left\{-2 \sum_{t \in T_{i}} \delta_{i t}(n) C_{r}^{t}\right\}\right) \\
& \quad \leq Q \leq \prod_{i=1}^{N}\left(1+2^{k} \exp \left\{-2 \sum_{t \in T_{i}} \delta_{i t}(n) C_{r}^{t}\right\}\right) .
\end{aligned}
$$

Condition (5) and the inequality $r>f(n)$ imply by (12) that

$$
2^{k} u<\varepsilon_{0} .
$$

Now we use bounds (19) and (20) and the elementary inequalities $1+u_{0} \leq e^{u_{0}}$ and $e^{u_{0}} \leq 1+2 u_{0}$. Then

$$
\prod_{i=1}^{N}\left(1-u_{i}\right) \geq 1-\sum_{i=1}^{N} u_{i}, \quad 0<u_{i}<1, i=0,1, \ldots, N
$$

and

$$
Q_{*} \leq Q \leq Q^{*}
$$

where

If

$$
Q^{*}=1+2^{k+1} u, \quad Q_{*}=1-2^{k} u \text {. }
$$

for some set $\left\{u_{1}, \ldots, u_{\nu}\right\}, 1 \leq u_{1}<\cdots<u_{\nu} \leq k, \nu=1, \ldots, k$, and some $t \in\{2, \ldots, r\}$, then inequality (10) holds, whence we get

$$
0 \leq i<r, \quad i \in I_{\left\{u_{1}, \ldots, u_{\nu}\right\}}, \quad 0 \leq j<r, \quad j \in J_{\left\{u_{1}, \ldots, u_{\nu}\right\}} .
$$

Applying the polynomial theorem and relation (21) we get

$$
2^{-k N}\left(2^{n k}-\sigma_{0}\right) Q_{*} \leq S_{1} \leq 2^{-k N}\left(2^{n k}-\sigma_{0}\right) Q^{*},
$$

where

$$
\sigma_{0}=1+\sum_{d=1}^{2^{k}-1} S_{d}^{(0)}(n, k ; 1)
$$

The definition of $S_{d}^{(0)}(n, k ; 1)$ differs from that of the term $S(n, k ; 1)$ in that the elements $i \in I$ and $j \in J$ on the right hand side of equality (8) satisfy an extra restriction, namely that there are exactly $d$ elements of the set

$$
\left\{\Gamma_{t, k}^{\left\{u_{1}, \ldots, u_{\nu}\right\}}, 1 \leq u_{1}<\cdots<u_{\nu} \leq k, \nu=1, \ldots, k\right\}
$$

for which relation (22) holds, $d=1,2, \ldots, 2^{k}-1$.

Let all the expressions

$$
\Gamma_{t, k}^{\left\{u_{1}, \ldots, u_{\nu}\right\}}, \quad 1 \leq u_{1}<\cdots<u_{\nu} \leq k, \nu=1, \ldots, k,
$$

be labeled with the numbers $1,2, \ldots, 2^{k}-1$. Assume that this numbering is a one-to-one correspondence between the expressions and the numbers. Then the sum $S_{d}^{(0)}(n, k ; 1)$ can be represented as follows:

$$
S_{d}^{(0)}(n, k ; 1)=\sum_{1 \leq \zeta_{1}<\cdots<\zeta_{d} \leq 2^{k}-1} S_{\left(\zeta_{1}, \ldots, \zeta_{d}\right)}^{(0)}(n, k ; 1),
$$


where the definition of $S_{\left(\zeta_{1}, \ldots, \zeta_{d}\right)}^{(0)}(n, k ; 1)$ differs from that of $S_{d}^{(0)}(n, k ; 1)$ by the restriction that relation (22) holds for those expressions $\Gamma_{t, k}^{\left\{u_{1}, \ldots, u_{\nu}\right\}}$ that correspond to the numbers $\zeta_{1}, \ldots, \zeta_{d}$. Denote by $A\left(\zeta_{1}, \ldots, \zeta_{d}\right)\left(B\left(\zeta_{1}, \ldots, \zeta_{d}\right)\right)$ the set of all $i \in I(j \in J)$ that are used in the bound (10) for all $\zeta_{1}, \ldots, \zeta_{d}$. By inequality (22), the number of elements of the set $A\left(\zeta_{1}, \ldots, \zeta_{d}\right)\left(B\left(\zeta_{1}, \ldots, \zeta_{d}\right)\right)$ is at least $2^{k-1}$ :

$$
\left|A\left(\zeta_{1}, \ldots, \zeta_{d}\right)\right| \geq 2^{k-1}, \quad\left|B\left(\zeta_{1}, \ldots, \zeta_{d}\right)\right| \geq 2^{k-1} .
$$

The sum $S_{d}^{(0)}(n, k ; 1)$ can be represented as follows:

$$
\begin{aligned}
& S_{d}^{(0)}(n, k ; 1) \\
& =\sum_{1 \leq \zeta_{1}<\cdots<\zeta_{d} \leq 2^{k}-1} \sum_{s=0}^{n-\rho n} C_{n-\rho n}^{s} \\
& \quad \times \sum_{s_{1}+s_{2}=s} C_{s}^{s_{1}}\left(\sum^{(1)} \frac{s_{1} !}{\prod_{i \in A} i !}\right)\left(\sum^{(2)} \frac{s_{2} !}{\prod_{i \in I \backslash A} i !}\right) \\
& \quad \times \sum_{s^{\prime}=0}^{\rho n} C_{\rho n}^{s^{\prime}} \sum_{s_{1}^{\prime}+s_{2}^{\prime}=s^{\prime}} C_{s^{\prime}}^{s_{1}^{\prime}}\left(\sum^{(3)} \frac{s_{1}^{\prime} !}{\prod_{j \in B} i !}\right)\left(\sum^{(4)} \frac{s_{2}^{\prime} !}{\prod_{j \in J \backslash B} i !}\right),
\end{aligned}
$$

where $\sum^{(1)}$ means the sum over all $i \in A\left(\zeta_{1}, \ldots, \zeta_{d}\right)$ such that $\sum i=s_{1}, \sum^{(2)}$ means the sum over all $i \in I \backslash A\left(\zeta_{1}, \ldots, \zeta_{d}\right)$ such that $\sum i=s_{2}, \sum^{(3)}$ means the sum over all $j \in$ $B\left(\zeta_{1}, \ldots, \zeta_{d}\right)$ such that $\sum j=s_{1}^{\prime}$, and $\sum^{(4)}$ means the sum over all $j \in J \backslash B\left(\zeta_{1}, \ldots, \zeta_{d}\right)$ such that $\sum j=s_{2}^{\prime}$.

Using the polynomial theorem and relations (25)-(27) we obtain the following bound:

$$
\begin{aligned}
\sigma_{0} \leq 1+2^{2^{k}-1} \sum_{s=0}^{n-\rho n} C_{n-\rho n}^{s}\left(2^{k-1}-1\right)^{s}\left(\sum_{s_{1} \geq 0} C_{s}^{s_{1}}\left(2^{k-1}\right)^{s_{1}}\right) \\
\times \sum_{s^{\prime}=0}^{\rho n} C_{\rho n}^{s^{\prime}}\left(2^{k-1}-1\right)^{s^{\prime}}\left(\sum_{s_{1}^{\prime} \geq 0} C_{s^{\prime}}^{s_{1}^{\prime}}\left(2^{k-1}\right)^{s_{1}^{\prime}}\right) .
\end{aligned}
$$

Taking into account the inequalities $s_{1} \leq\left[r 2^{k}\right]$ and $s_{1}^{\prime} \leq\left[r 2^{k}\right]$, we conclude from (28) that

$$
\sigma_{0} \leq 2^{2^{k}}\left(2^{k-1}\right)^{n}\left(2^{k}\right)^{r 2^{k}}\left(\sum_{s_{1}=0}^{\left[r 2^{k}\right]} C_{n-\rho n}^{s_{1}}\right)\left(\sum_{s_{1}^{\prime}=0}^{\left[r 2^{k}\right]} C_{\rho n}^{s_{1}^{\prime}}\right) .
$$

This implies

$$
\sigma_{0} \leq 2^{2^{k}}\left(2^{k-1}\right)^{n}\left(2^{k}\right)^{r 2^{k}}\left(r 2^{k}\right)^{2}\left(C_{n}^{\left[r 2^{k}\right]}\right)^{2}
$$

Then

$$
0 \leq \sigma_{0} \leq 2^{n k} \Theta_{2}
$$

by inequality (29), condition (12), and by the lower bound

$$
n !>n^{n} e^{-n} \sqrt{2 \pi n} e^{1 /(12 n+1)}
$$

proved in [2]. 
Considering condition (3) and relations (21), (24), and (30), we get the following bounds:

$$
\begin{aligned}
\lambda^{k}- & \left\{2^{(m+1) k} u+2^{m k} \Theta_{2}\left(1+2^{k} u\right)\right\} \\
& \leq S_{1} \leq \lambda^{k}+\left\{2^{(m+1) k+1} u+2^{m k} \Theta_{2}\left(1+2^{k+1} u\right)\right\} .
\end{aligned}
$$

Using restrictions (12) we show that

$$
p_{1} \leq 7\left(2^{2^{k}}\right) 2^{(m+1) k} \exp \left\{-2^{-2 k} \sum_{i=1}^{N} \delta_{i}+\ln (\tilde{\rho} n)-m \ln 2\right\}
$$

for $\Delta \geq 1$. Indeed, put

$$
p_{2}=p_{1}-S_{2}
$$

where

$$
S_{2}=2^{-k N} \sum_{\Delta=1}^{2^{k}-1} S_{\left(G_{0}\right)}^{(\Delta)}(n, k ; Q)
$$

The definition of $S_{\left(G_{0}\right)}^{(\Delta)}(n, k ; Q)$ differs from that of the term $S^{(\Delta)}(n, k ; Q)$ in that the index of summation $s^{\prime}$ in (8) satisfies the additional condition, called $G_{0}$ :

$$
\rho n-r+1 \leq s^{\prime} \leq \rho n
$$

Now we find a bound for $S_{2}$. Denote by $M_{1}\left(\tilde{M}_{1}\right)$ the family of all $i \in I(j \in J)$ that do not belong to $I_{\omega_{\alpha}}\left(J_{\omega_{\alpha}}\right), \alpha=1, \ldots, \Delta$. Also we put

$$
M_{2}=I \backslash M_{1}, \quad \tilde{M}_{2}=J \backslash \tilde{M}_{1} .
$$

Let $R_{1}\left(\tilde{R}_{1}\right)$ denote the number of elements of the set $M_{1}\left(\tilde{M}_{1}\right)$. Let $z$ be the minimum number such that

$$
\Delta \leq 2^{z}-1, \quad 1 \leq z \leq k
$$

According to Proposition 2.1 of [1] we obtain

$$
R_{1} \leq 2^{k-z}-1, \quad \tilde{R}_{1} \leq 2^{k-z}-1 .
$$

If lower bound (17) holds, we take into account (4) and get the following inequality for $Q$ defined in 34 :

$$
Q \leq 2^{z N}\left(1+2^{-z}\left(2^{k}-\Delta-1\right) u\right) .
$$

Relation (16) implies that

$$
0 \leq i<r \quad(0 \leq j<r)
$$

for all $i \in M_{2}\left(j \in \tilde{M}_{2}\right)$ by condition (22) and (23). Using (36)-(38) and condition $G_{0}$, we prove that

$$
S_{2} \leq 2^{2^{k}} 2^{(k-1) m} \exp \left\{-\rho n 2^{-k}+2^{k} \varepsilon \ln ^{2} n \ln \left(\frac{\tilde{\rho} n e}{2^{k} \varepsilon \ln ^{2} n}\right)+2^{k} u\right\} .
$$

Now we introduce condition $G_{1}$ : let

$$
s^{\prime} \leq \rho n-r
$$

and let there exist $i \in M_{2}$ and (or) $j \in \tilde{M}_{2}$ such that $i \in\left(r / E_{n}, r\right]$ and (or) $j \in\left(r / E_{n}, r\right]$, where

$$
E_{n}>3, \quad E_{n}=o(\ln n), \quad n \rightarrow \infty .
$$

Let

$$
p_{3}=p_{2}-S_{3}
$$


where

$$
S_{3}=2^{-k N} \sum_{\Delta=1}^{2^{k}-1} S_{\left(G_{1}\right)}^{(\Delta)}(n, k ; Q) .
$$

The definition of $S_{\left(G_{1}\right)}^{(\Delta)}(n, k ; Q)$ differs from that of the term $S^{(\Delta)}(n, k ; Q)$ in that the index of summation $s^{\prime}$ in (8) satisfies the additional condition $G_{1}$.

We show that

$$
S_{3} \leq \frac{2^{2^{k}} 2^{m k}}{2^{m}} \exp \left\{-2^{-k} N\left(1-N^{-A_{n}}\right)+2^{k} \varepsilon \ln ^{2} n \ln \left(\frac{\tilde{\rho} n e}{2^{k} \varepsilon \ln ^{2} n}\right)\right\}
$$

where $A_{n}=2 \varepsilon / E_{n}$. If condition $G_{1}$ holds, we get

$$
\Gamma_{t, k}^{\omega_{\alpha}} \geq C_{r}^{t-1} \frac{r}{E_{n}}
$$

for all $t \in\{2, \ldots, r\}$ and some $\alpha=1, \ldots, \Delta$ by inequality (11). Using bound (42) and condition (4) we obtain

$$
\left|\prod_{t=1}^{g_{i}(n)}\left(1-2 p_{i t}\right)^{\Gamma_{t, k}^{\omega_{\alpha}}}\right| \leq \exp \left\{-2 \sum_{t \in T_{i}} \delta_{i t}(n) C_{r}^{t-1} \frac{r}{E_{n}}\right\}, \quad i=1, \ldots, N .
$$

The latter bound implies

$$
Q \leq 2^{z N} \exp \left\{-2^{-z}\left(N-\sum_{i=1}^{N} \exp \left\{-2 \sum_{t \in T_{i}} \delta_{i t}(n) C_{r}^{t-1} \frac{r}{E_{n}}\right\}\right)\right\} .
$$

Relation (43) yields

$$
Q \leq \hat{Q}
$$

by Hölder's inequality and relation (20), where

$$
\hat{Q}=2^{z N} \exp \left\{-2^{-z}\left(N-N^{1-A_{n}}\right)\right\} .
$$

Now we derive from condition $G_{1}$ that

$$
\begin{aligned}
S_{3} \leq & 2^{2^{k}} \sum_{s=0}^{n-\rho n} C_{n-\rho n}^{s} \sum_{s_{1}+s_{2}=s} C_{s}^{s_{1}}\left(\sum_{\sum_{i \in M_{2}} i=s_{1}} \frac{s_{1} !}{\prod_{i \in M_{2}} i !}\right)\left(\sum_{\sum_{i \in M_{1}} i=s_{2}} \frac{s_{2} !}{\prod_{i \in M_{1}} i !}\right) \\
& \times \sum_{s^{\prime}=0}^{\rho n-r} C_{\rho n}^{s^{\prime}} \sum_{s_{1}^{\prime}+s_{2}^{\prime}=s^{\prime}} C_{s^{\prime}}^{s_{1}^{\prime}}\left(\sum_{\sum_{j \in \tilde{M}_{2}} j=s_{1}^{\prime}} \frac{s_{1}^{\prime} !}{\prod_{j \in \tilde{M}_{2}} j !}\right)\left(\sum_{\sum_{j \in \tilde{M}_{1} j=s_{2}^{\prime}}} \frac{s_{2}^{\prime} !}{\prod_{j \in \tilde{M}_{1}} j !}\right) Q .
\end{aligned}
$$

Relations (36), (38), (44), and (45) prove (41).

Now we introduce condition $G_{2}$ : let inequality (40) hold and let there exist $i \in M_{2}$ and (or) $j \in \tilde{M}_{2}$ such that $i \in\left(r / \ln n, r / E_{n}\right]$ and (or) $j \in\left(r / \ln n, r / E_{n}\right]$.

Put

$$
p_{4}=p_{3}-S_{4},
$$

where

$$
S_{4}=2^{-k N} \sum_{\Delta=1}^{2^{k}-1} S_{\left(G_{2}\right)}^{(\Delta)}(n, k ; Q)
$$

The definition of $S_{\left(G_{2}\right)}^{(\Delta)}(n, k ; Q)$ differs from that of the term $S^{(\Delta)}(n, k ; Q)$ in that the index of summation $s^{\prime}$ in the sum (8) satisfies condition $G_{2}$. 
We show that

$$
S_{4} \leq \frac{2^{2^{k}} 2^{m k}}{2^{m}} \exp \left\{-2^{-k}\left(1-e^{-2 \varepsilon}\right) N+\frac{2^{k} \varepsilon \ln ^{2} n}{E_{n}} \ln \left(\frac{\tilde{\rho} n e E_{n}}{2^{k} \varepsilon \ln ^{2} n}\right)\right\}
$$

Similarly to the proof of (44) we obtain

$$
Q \leq 2^{z N} \exp \left\{-2^{-k}\left(1-e^{-2 \varepsilon}\right) N\right\}
$$

if condition $G_{2}$ holds. Note that the constant $\tilde{A}_{n}=2 \varepsilon / \ln n$ substitutes the constant $A_{n}=2 \varepsilon / E_{n}$ in the proof.

If the indices $i$ and $j$ satisfy condition $G_{2}$, then bound (46) follows from (36) and (47) similarly to the proof of the corresponding bound for $S_{3}$.

The following condition is called $G_{3}$ : let inequality (40) hold and let

$$
0 \leq i \leq \frac{r}{\ln n} \quad \text { and } \quad 0 \leq j \leq \frac{r}{\ln n}
$$

for all $i \in M_{2}$ and $j \in \tilde{M}_{2}$. Put

$$
p_{5}=p_{4}-S_{5},
$$

where

$$
S_{5}=2^{-k N} \sum_{\Delta=1}^{2^{k}-1} S_{\left(G_{3}, 2^{z}-2\right)}^{(\Delta)}(n, k ; Q) .
$$

The definition of $S_{\left(G_{3}, 2^{z}-2\right)}^{(\Delta)}(n, k ; Q)$ differs from that of the term $S^{(\Delta)}(n, k ; Q)$ in that the index of summation $s^{\prime}$ in (8) satisfies condition $G_{3}$ and that $\Delta<2^{z}-1$.

We show that

$$
S_{5} \leq \frac{2^{2^{k}} 2^{m k}}{2^{m}} \exp \left\{-2^{-k} N+2^{k} \varepsilon \ln ^{2} n+2^{k} u\right\} .
$$

Using (40) and inequality (10), we get

$$
\Gamma_{t, k}^{\omega_{\alpha}} \geq C_{r}^{t-1}\left(s^{(\alpha)}+\tilde{s}^{(\alpha)}\right)
$$

for all $t \in\{2, \ldots, r\}$ and $\alpha=1, \ldots, \Delta$, where

$$
s^{(\alpha)}=\sum_{i \in I_{\omega_{\alpha}}} i, \quad \tilde{s}^{(\alpha)}=\sum_{j \in J_{\omega_{\alpha}}} j .
$$

According to (4),

$$
\left|\prod_{t=1}^{g_{i}(n)}\left(1-2 p_{i t}\right)^{\Gamma_{t, k}^{\omega_{\alpha}}}\right| \leq \prod_{t \in T_{i}}\left(1-2 \delta_{i t}(n)\right)^{\Gamma_{t, k}^{\omega_{\alpha}}}
$$

for $i=1, \ldots, N$ and $\alpha=1, \ldots, \Delta$.

Now we apply (51) to the right hand side of (52). Then

$$
\prod_{t \in T_{i}}\left(1-2 \delta_{i t}(n)\right)^{\Gamma_{t, k}^{\omega_{\alpha}}} \leq \exp \left\{-\frac{2 \delta_{i}}{\sqrt[6]{n}}\left(s^{(\alpha)}+\tilde{s}^{(\alpha)}\right)\right\} .
$$

The inequality $e^{-y} \leq 1-y / 2,0 \leq y<1$, implies that

$$
\exp \left\{-\frac{2 \delta_{i}}{\sqrt[6]{n}}\left(s^{(\alpha)}+\tilde{s}^{(\alpha)}\right)\right\} \leq 1-\frac{\delta_{i}}{\sqrt[6]{n}}\left(s^{(\alpha)}+\tilde{s}^{(\alpha)}\right)
$$


for $i=1, \ldots, N$ and $\alpha=1, \ldots, \Delta$. In turn, inequality (54) yields

$$
\begin{aligned}
& 2^{-k N} \sum_{\Delta=1}^{2^{k}-1} S_{\left(G_{3}\right)}^{(\Delta)}(n, k ; Q) \\
& \leq 2^{-k N} 2^{2^{k}}(\Delta+1)^{N} \\
& \times \sum_{s=0}^{n-\rho n} C_{n-\rho n}^{s} \sum_{s_{*}=0}^{s} R_{1}^{s-s_{*}}\left(\sum_{\sum_{i \in M_{2}} i=s_{*}} \frac{s !}{\left(s-s_{*}\right) !}\left(\prod_{i \in M_{2}} i !\right)^{-1}\right) \\
& \times \sum_{s^{\prime}=0}^{\rho n} C_{\rho n}^{s^{\prime}} \sum_{\tilde{s}_{*}=0}^{s^{\prime}} \tilde{R}_{1}^{s^{\prime}-\tilde{s}_{*}}\left(\sum_{\sum_{i \in \tilde{M}_{2}} i=\tilde{s}_{*}} \frac{s !}{\left(s^{\prime}-\tilde{s}_{*}\right) !}\left(\prod_{j \in \tilde{M}_{2}} j !\right)^{-1}\right) \\
& \times \exp \left\{-2^{-z} \sum_{i=1}^{N} \frac{\delta_{i}}{\sqrt[6]{n}} \sum_{\alpha=1}^{\Delta}\left(s^{(\alpha)}+\tilde{s}^{(\alpha)}\right)+2^{k} u\right\}, \quad s+s^{\prime} \geq 1,
\end{aligned}
$$

where the definition of $S_{\left(G_{3}\right)}^{(\Delta)}(n, k ; Q)$ differs from that of $S^{(\Delta)}(n, k ; Q)$ in the restriction that the indices in the sum (8) satisfy condition $G_{3}$.

If $\Delta<2^{z}-1$, then (55) implies bound (50) by condition (3), inequalities (36),

$$
\max \left\{s_{*}, \tilde{s}_{*}\right\} \leq 2^{k} \varepsilon \ln n,
$$

and

$$
\sum_{\alpha=1}^{\Delta}\left(s^{(\alpha)}+\tilde{s}^{(\alpha)}\right) \geq s_{*}+\tilde{s}_{*}
$$

where

$$
s_{*}=\sum_{i \in M_{2}} i, \quad \tilde{s}_{*}=\sum_{j \in \tilde{M}_{2}} j .
$$

Now let $\Delta=2^{z}-1$. Put

$$
p_{6}=p_{5}-S_{6},
$$

where

$$
S_{6}=2^{-k N} \sum_{\Delta=1}^{2^{k}-1} S_{\left(G_{3}, 2^{z}-1\right)}^{(\Delta)}(n, k ; Q) .
$$

The definition of $S_{\left(G_{3}, 2^{z}-1\right)}^{(\Delta)}(n, k ; Q)$ differs from that of the term $S^{(\Delta)}(n, k ; Q)$ in that the index of summation $s^{\prime}$ in (8) satisfies condition $G_{3}$ and $\Delta=2^{z}-1$. If condition $G_{3}$ holds and $\Delta=2^{z}-1$, then we use condition (31) and relations (36) and (55) together with inequality (56) to find a bound for $S_{6}$ :

$$
S_{6} \leq 2^{2^{k}} 2^{m k} \exp \left\{-2^{-2 k} \sum_{i=1}^{N} \delta_{i}+k+\ln (\tilde{\rho} n)-m \ln 2\right\}
$$

for the case of

$$
s_{*}+\tilde{s}_{*} \geq 1 .
$$

Now we show that there exists $\alpha \in\{1,2, \ldots, \Delta\}$ such that $\xi_{\alpha} \leq 2$ if $\Delta=2^{z}-1$, $1 \leq z \leq k$, and either $z \in\{k, k-1\}$ or $k \in\{1,2\}$. Indeed, if either $z=k$ or $k \in\{1,2\}$, then this property is obvious. If $z=k-1$, then we derive this property from Remark 2.2 in [1]. 
Consider condition $G_{4}$ : let inequality (40) hold and let

$$
\begin{gathered}
s_{*}+\tilde{s}_{*}=0, \\
\xi_{\alpha} \geq 3, \quad \alpha=1, \ldots, \Delta, \Delta=2^{z}-1,1 \leq z \leq k-2,3 \leq k<\infty .
\end{gathered}
$$

Put

$$
p_{7}=p_{6}-S_{7}
$$

and

$$
S_{7}=2^{-k N} \sum_{\Delta=1}^{2^{k}-1} S_{\left(G_{4}\right)}^{(\Delta)}(n, k ; Q),
$$

where the definition of $S_{\left(G_{4}\right)}^{(\Delta)}(n, k ; Q)$ differs from that of the term $S^{(\Delta)}(n, k ; Q)$ in that the index of summation $s^{\prime}$ in (8) satisfies conditions $G_{4}$.

Now we obtain a bound for $S_{7}$. If (59) holds, $\Delta=2^{z}-1$, and $\tilde{R}_{1}<2^{k-z}-1$, then we rewrite bound (55) as follows:

$$
\begin{aligned}
S_{7} & \leq 2^{2^{k}+1+z N-k N} \sum_{s=0}^{n-\rho n} C_{n-\rho n}^{s}\left|M_{1}\right|^{s} \sum_{\substack{s^{\prime}=0 \\
s^{\prime}+s \geq 1}}^{\rho n} C_{\rho n}^{s^{\prime}}\left|\tilde{M}_{1}\right|^{s^{\prime}} \\
& \leq \frac{2^{2^{k}+1+m k}}{2^{m}}\left(1-2^{1-k}\right)^{\rho n}
\end{aligned}
$$

(here we used restrictions (36)).

It remains to check that

$$
S_{8} \leq \frac{2^{2^{k}} 2^{m k}}{2^{m}} \exp \left\{-\rho n 2^{-k+1}+\varepsilon \ln ^{2} n \ln \left(\frac{\rho n e}{\varepsilon \ln ^{2} n}\right)\right\}
$$

if the conditions $G_{4}$ hold and

$$
\tilde{R}_{1}=2^{k-z}-1
$$

where

$$
p_{7}=S_{8}=2^{-k N} \sum_{\Delta=1}^{2^{k}-1} S_{\left(G_{4}, \tilde{R}_{1}\right)}^{(\Delta)}(n, k ; Q) .
$$

Here the definition of $S_{\left(G_{4}, \tilde{R}_{1}\right)}^{(\Delta)}(n, k ; Q)$ differs from that of the term $S^{(\Delta)}(n, k ; Q)$ in that the index of summation $s^{\prime}$ in (8) satisfies conditions $G_{4}$ and (63).

Similarly to the proof in [1] and according to Proposition 2.2 in [1] we conclude from (63) and $G_{4}$ that there exists at least one element $j_{*} \in \tilde{M}_{1}$ such that $j_{*} \leq r$. Therefore, under the assumptions of the theorem and conditions $G_{4}$ and (63), we get

$$
\begin{aligned}
S_{8} & \leq 2^{2^{k}} 2^{-k N} 2^{z N} 2^{(k-z)(n-\rho n)} \sum_{\substack{s^{\prime}=0 \\
s+s^{\prime} \geq 1}}^{\rho n-r} C_{\rho n}^{s^{\prime}} \sum_{\sum_{j \in \tilde{M}_{1}} j=s^{\prime}} \frac{s^{\prime} !}{\prod_{j \in \tilde{M}_{1}} j !} \\
& =2^{2^{k}} 2^{-k N} 2^{z N+(k-z)(n-\rho n)} \sum_{\substack{s^{\prime}=0 \\
s+s^{\prime} \geq 1}}^{\substack{\rho n-r \\
\rho n}} \sum_{\substack{s^{\prime} \\
s_{1}^{\prime}+j_{*}=s^{\prime} \\
j_{*} \leq r}} C_{s^{\prime}}^{s^{\prime}{ }^{\prime}}\left(\sum_{\sum_{j \in \tilde{M}_{1} \backslash j_{*}} j=s_{1}^{\prime}} \frac{s_{1}^{\prime} !}{\prod_{j \in \tilde{M}_{1} \backslash j_{*}} j !}\right) \\
& \leq 2^{2^{k}} 2^{(k-z) m}\left(1-\frac{1}{2^{k-z}}\right)^{\rho n} \sum_{j_{*}=0}^{r} C_{\rho n}^{j_{*} .}
\end{aligned}
$$

Applying inequality (31) we prove bound (62). 
Considering conditions $G_{0}-G_{4}$, we make sure that they exhaust all possible cases of summation in (8) with respect to the parameters $s, s^{\prime}, i, j, i \in I$ and $j \in J$ for which inequality (16) holds if $\Delta \geq 1$.

Therefore relations (39), (41), (46), (50), (57), (61), and (62) prove (33) under the assumptions of the lemma. Then, by (18), (32), and (33), we find that $\mathrm{E} \nu_{n}^{[k]}=\lambda^{k}+$ $\Delta(k, n)$, where

$$
\begin{gathered}
\Delta(k, n)=\psi(k, n)+p_{1}, \\
-\left\{2^{(m+1) k} u+2^{m k} \Theta_{2}\left(1+2^{k} u\right)\right\} \\
\leq \psi(k, n) \leq 2^{(m+1) k+1} u+2^{m k} \Theta_{2}\left(1+2^{k+1} u\right) .
\end{gathered}
$$

Using relations (33) and (65), we complete the proof of (13) and (14).

\section{Proof of the theorem}

Fix an integer $q \geq 0$. Consider the following inequality:

$$
\left|\mathrm{P}\left\{\nu_{n}=q\right\}-\frac{\lambda^{q}}{q !} e^{-\lambda}\right| \leq R_{1}+R_{2}+R_{3}
$$

where

$$
\begin{aligned}
& R_{1}=\left|\mathrm{P}\left\{\nu_{n}=q\right\}-\sum_{k=q}^{q+2 \nu-1}(-1)^{k-q} C_{k}^{q} B_{k n}\right|, \\
& R_{2}=\left|\sum_{k=q}^{q+2 \nu-1}(-1)^{k-q} C_{k}^{q}\left\{B_{k n}-\frac{\lambda^{k}}{k !}\right\}\right|, \\
& R_{3}=\left|\sum_{k=q}^{q+2 \nu-1}(-1)^{k-q} C_{k}^{q} \frac{\lambda^{k}}{k !}-\frac{\lambda^{q}}{q !} e^{-\lambda}\right|,
\end{aligned}
$$

and $B_{k n}$ denotes the binomial moment of order $k$ for the random variable $\nu_{n}$. Choose $n$ such that

$$
\frac{\lambda^{q+2 \nu}}{q !(2 \nu) !}<\left(\frac{2 e \lambda}{\gamma}\right)^{\gamma}
$$

where $2 \nu=\gamma-q \geq 0$. Such a number $n$ exists in view of $n \geq 2^{6 q}$. The inequality

$$
R_{3}<\frac{\lambda^{q+2 \nu}}{q !(2 \nu) !}
$$

together with (67) implies that

$$
R_{3}<\left(\frac{2 e \lambda}{\gamma}\right)^{\gamma}
$$


Applying (13) we prove that

$$
\begin{aligned}
&\left|B_{q+2 \nu, n}-\frac{\lambda^{q+2 \nu}}{(q+2 \nu) !}\right| \\
&=\frac{1}{(q+2 \nu) !}|\Delta(q+2 \nu, n)| \\
& \leq \frac{2^{(q+2 \nu) m}}{(q+2 \nu) !}\left(2^{q+2 \nu+1} B(n)+\Theta_{2}\left(1+2^{q+2 \nu+1} B(n)\right)\right) \\
&+\frac{2^{(q+2 \nu) m}}{(q+2 \nu) !}\left(7 \exp \left\{-2^{-2 \gamma} \sum_{i=1}^{N} \delta_{i}+(q+2 \nu)+\sqrt[6]{n}+\ln (\tilde{\rho} n)-m \ln 2\right\}\right) .
\end{aligned}
$$

Taking into account (12), we get

$$
\left|B_{q+2 \nu, n}-\frac{\lambda^{q+2 \nu}}{(q+2 \nu) !}\right| \leq \frac{2^{m \gamma}}{\gamma !}\left(2^{\gamma+1} B(n)+\Theta_{2}\left(1+2^{\gamma+1} B(n)\right)+7 \Theta_{1}\right)
$$

By Bonferroni's inequality $[\underline{3}$,

$$
0 \leq \mathrm{P}\left\{\nu_{n}=q\right\}-\sum_{k=q}^{q+2 \nu-1}(-1)^{k-q} C_{k}^{q} B_{k n} \leq C_{q+2 \nu}^{q} B_{q+2 \nu, n} .
$$

Using (67) and (72), we derive from (71) that

$$
R_{1}<\left(\frac{2 e \lambda}{\gamma}\right)^{\gamma}\left(1+2^{\gamma+1} B(n)+\Theta_{2}\left(1+2^{\gamma+1} B(n)\right)+7 \Theta_{1}\right)
$$

Consider

$$
R_{2}=\left|\sum_{k=q}^{q+2 \nu-1}(-1)^{k-q} C_{k}^{q}\left[B_{k n}-\frac{\lambda^{k}}{k !}\right]\right|
$$

It is easy to show that

$$
\sup _{q \leq k \leq q+2 \nu-1} C_{k}^{q}\left|B_{k n}-\frac{\lambda^{k}}{k !}\right| \leq e^{4 \lambda} B(n)+e^{2 \lambda}\left(\Theta_{2}\left(1+2^{\gamma+1} B(n)\right)+7 \Theta_{1}\right)
$$

by (12)-(14). Inequality (74) implies

$$
R_{2}<\sum_{k=q}^{q+2 \nu-1} C_{k}^{q}\left|B_{k n}-\frac{\lambda^{k}}{k !}\right| \leq e^{4 \lambda} \gamma B(n)+e^{2 \lambda} \gamma\left(\Theta_{2}\left(1+2^{\gamma+1} B(n)\right)+7 \Theta_{1}\right)
$$

Thus (66), (69), (733), and (75) imply (6). The theorem is proved. 


\section{BIBLIOGRAPHY}

1. V. I. Masol, A theorem on the limit distribution of the number of false solutions of a system of nonlinear random Boolean equations, Teor. Veroyatnost. i Primenen. 43 (1998), no. 1, 41-56; English transl. in Theory Probab. Appl. 43 (1999), no. 1, 75-88. MR.1669972 (2000f:60040)

2. W. Feller, An Introduction to Probability Theory and its Applications, 3rd ed., vol. I, John Wiley \& Sons, New York-London-Sydney, 1968. MR0228020 (37:3604)

3. V. N. Sachkov, Introduction to Combinatorial Methods of Discrete Mathematics, "Nauka", Moscow, 1982. (Russian) MR700691 (85g:05001)

Department of Probability Theory and Mathematical Statistics, Faculty for Mechanics and Mathematics, National Taras Shevchenko University, Academician Glushkov Avenue 6 , KYIV 03127, UKRAINE

E-mail address: vimasol@ukr.net

Department of Probability Theory and Mathematical Statistics, Faculty for Mechanics and Mathematics, National Taras Shevchenko University, Academician Glushkov Avenue 6, KYIV 03127, UKRAINE

E-mail address: mslob@ukr.net

Received 10/FEB/2006

Translated by S. KVASKO 